Juliana M. Kling, MD, MPH, NCMP, FACP

Associate Professor of Medicine and Interim Chair and

Associate Chair for Research in Women's Health Internal

Medicine, Associate Chair of Equity, Inclusion and Diversity,

Department of Medicine, Mayo Clinic Arizona, Scottsdale
Sharon J. Parish, MD

Professor of Medicine in Clinical Psychiatry and Professor

of Clinical Medicine, Weill Cornell Medical College,

Department of Psychiatry and Department of Medicine,

New York, NY

\title{
Trials and tribulations of testosterone therapy in women: Importance of adhering to the evidence
}

\section{Testosterone \\ should not \\ be touted \\ for improving \\ energy, \\ well-being, \\ or other \\ outcomes \\ not supported \\ by data}

\begin{abstract}
Sexual health is an important part of $\checkmark$ women's lives. When sexual function is impaired, it can negatively affect quality of life, self-image, and relationship satisfaction and lead to poorer health outcomes. ${ }^{1,2}$ As many as $43.1 \%$ of women of all ages report a sexual problem, with $12 \%$ of women having or reporting a distressing sexual problem. ${ }^{2} \mathrm{~A}$ biopsychosocial approach is ideal for evaluating, diagnosing, and treating women who have sexual dysfunction. ${ }^{3}$
\end{abstract}

See related article, page 35

Testosterone treatment has been found to be effective in select postmenopausal women who are diagnosed with hypoactive sexual desire disorder (HSDD), which is characterized by low sexual desire causing distress. ${ }^{4} \mathrm{How}$ ever, no US Food and Drug Administration (FDA)-approved formulation of testosterone is available for women in the United States, due to its unknown long-term safety.

This has not stopped many physicians and other medical practitioners from prescribing custom-compounded or dose-reduced male products to women. ${ }^{5}$ Some prescribers have shared partial truths through Internet-based marketing to support "bioidentical" testosterone as a "natural" hormone, free of risk and with unfounded benefits, ${ }^{6}$ making it difficult for women to know whom to trust and where to go regarding their sexual health concerns.

In this issue of Cleveland Clinic Journal of Medicine, Smith and Batur ${ }^{7}$ provide a comprehensive review of the effects of androgens doi:10.3949/ccjm.88a.20195 in women, as well as clinically relevant bestpractice recommendations for the use of androgens in women. They discuss the different types of endogenous androgens and how they interact with various biologic systems in women. They appropriately highlight how a state of "female androgen insufficiency" has not been established in women, such as exists for men, as low serum androgen levels do not reliably correlate with symptoms such as chronic fatigue or decreased mood in women. They also discuss the difficulty of accurately measuring testosterone levels, including pointing out that salivary testing is not recommended for clinical use.

\section{LABELS MATTER: DESIRE VS AROUSAL}

Nomenclature from the Diagnostic and Statistical Manual of Mental Disorders, 5th Edition specifies the category of female sexual dysfunction called female sexual interest and arousal disorder (FSIAD), ${ }^{8}$ which may be useful for psychiatry or behavioral health practitioners, but does not have support from clinical data nor practicality in the clinical settings in which practitioners manage sexual problems.

Based on the published evidence, reputable organizations such as the Fourth International Consultation on Sexual Medicine and the International Society for the Study of Women's Sexual Health have clearly stated that desire and arousal are distinct conditions. Thus, the nomenclature and diagnostic criteria for HSDD and FSIAD must remain separate when diagnosing, treating, and coding female sexual dysfunction. ${ }^{9,10}$ In 
fact, the female sexual health outcome evaluated in the studies demonstrating efficacy of testosterone in postmenopausal women was not FSIAD, but rather sexual satisfaction, sexual desire, or HSDD.

\section{NEW PUBLICATIONS}

Smith and Batur's review is timely and summarizes some important information published over the last year. For example, a 2019 systematic review and meta-analysis of randomized controlled trial data found that transdermal testosterone is effective for postmenopausal women with low sexual desire causing distress, with few short-term side effects (acne and hair growth), particularly when dosed in the normal physiologic range for premenopausal women. ${ }^{5}$

Further, they point out that there are not enough data to let us say that testosterone treatment is associated with improved individual well-being, cognitive health, or musculoskeletal outcomes, claims that are commonly endorsed by custom-compounding bioidentical providers.

Recently, the Global Consensus Position Statement on the Use of Testosterone Therapy for Women was published and endorsed by many respected US and international organizations such as the International Menopause Society, the Endocrine Society, the North American Menopause Society, and the International Society for the Study of Women's Sexual Health. ${ }^{11}$ One of its evidence-based and expert-supported recommendations is that the only indication for the use of testosterone in women is for treatment of postmenopausal women who have been diagnosed with HSDD and that the dosage should approximate a normal physiologic level of testosterone for a premenopausal woman.

Importantly, the Global Consensus Position Statement recommends against compounded bioidentical testosterone therapy in view of lack of evidence of efficacy and safety, and cautioned against testosterone pellets or injections, which typically result in supraphysiologic concentrations. Those levels may lead to long-term, irreversible side effects in- cluding voice changes and clitoromegaly.

Consistent with this recommendation, the National Academies of Sciences, Engineering, and Medicine ${ }^{12}$ recently published a review and called for custom-compounded bioidentical hormones to be listed as "difficult to compound" as a first step to improving quality control. They recommended that state medical boards of pharmacy expand and improve oversight and standardization of these products due to clinical concerns regarding the safety and effectiveness of custom-compounded bioidentical hormones.

With more and more FDA-approved bioidentical hormone products available, healthcare practitioners have access to a wealth of safe and effective treatments for their female patients. Hopefully, with growing clarity regarding the evidence of safety and efficacy of testosterone in women with HSDD, a testosterone formulation for women will join the list of FDA-approved products.

\section{ALIGNING PRACTICE WITH EVIDENCE}

It is important for physicians and other healthcare practitioners caring for women to ensure that their practice aligns with the evidence regarding testosterone treatment for women. There is a great need for comprehensive sexual health treatment, given the significant numbers of women suffering from sexual problems. For those select women with HSDD, transdermal testosterone, properly dosed and monitored, can provide important improvements in quality of life and sexual function.

Testosterone should not be touted as a treatment for improving energy, well-being, or other outcomes that are not supported by data, especially with formulations that put women at risk of supraphysiologic levels. Smith and Batur's review provides a clinically relevant and valuable summary to communicate this important message.

\section{DISCLOSURES}

Dr. Parish has disclosed membership on advisory committees or review panels for AMAG and consulting for Strategic Science Technologies. Dr. Kling reports no relevant financial relationships which, in the context of her contribution, could be perceived as a potential conflict of interest.

\section{Dosage should be in the physiologic range for a premenopausal woman}

a




\section{REFERENCES}

1. Biddle AK, West SL, D'Aloisio AA, Wheeler SB, Borisov NN, Thorp J. Hypoactive sexual desire disorder in postmenopausal women: quality of life and health burden. Value Health 2009; 12(5):763-772 doi:10.1111/j.1524-4733.2008.00483.x

2. Shifren JL, Monz BU, Russo PA, Segreti A, Johannes CB. Sexual problems and distress in United States women: prevalence and correlates. Obstet Gynecol 2008; 112(5):970-978.

doi:10.1097/AOG.0b013e3181898cdb

3. Thomas HN, Thurston RC. A biopsychosocial approach to women's sexual function and dysfunction at midlife: a narrative review. Maturitas 2016; 87:49-60. doi:10.1016/j.maturitas.2016.02.009

4. Islam RM, Bell RJ, Green S, Page MJ, Davis SR. Safety and efficacy of testosterone for women: a systemic review and meta-analysis of randomized controlled trial data. Lancet Diabetes Endocrinol 2019; 7(10):754-766. doi:10.1016/S2213-8587(19)30189-5

5. Vegunta S, Kling JM, Kapoor E. Androgen therapy in women. J Women's Health 2020; 29(1):57-64. doi:10.1089/jwh.2018.7494

6. Liss J, Santoro N. Stand firm with science. Contemp OB/GYN 2020; 65(10):17-19.

7. Smith T, Batur P. Prescribing testosterone and DHEA: the role of androgens in women. Cleve Clin J Med 2021; 88(1):35-43. doi:10.3949/ccjm.88a.20030

8. Diagnostic and Statistical Manual of Mental Disorders, 5th ed. Arlington, VA: American Psychiatric Association, 2013.

9. McCabe MP, Sharlip ID, Atalla E, et al. Definitions of sexual dysfunction in women and men: a consensus statement from the Fourth International Consultation on Sexual Medicine 2015. J Sex Med 2016; 13(2):135-143. doi:10.1016/j.jsxm.2015.12.019

10. Parish SJ, Goldstein AT, Goldstein SW, et al. Toward a more evidencebased nosology and nomenclature for female sexual dysfunctions Part II. J Sex Med 2016; 13(12):1888-1906. doi:10.1016/j.jsxm.2016.09.020

11. Davis SR, Baber R, Panay N, et al. Global consensus statement on the use of testosterone therapy for women. Climacteric 2019; 22(5):429434. doi:10.1080/13697137.2019.1637079

12. National Academies of Sciences, Engineering, and Medicine 2020. The Clinical Utility of Compounded Bioidentical Hormone Therapy: A Review of Safety, Effectiveness, and Use. Washington, DC: The National Academies Press. doi:10.17226/25791

Address: Juliana Kling, MD, MPH, NCMP, FACP, Mayo Clinic Arizona, 13737 North 92nd Street, Scottsdale, AZ 85260; Kling.Juliana@mayo.edu 